\title{
A Case of Acquired von Willebrand Syndrome Complicated by Acute Myelomonocytic Leukemia
}

\author{
Masahiko Fukatsu $^{a}$ Hiroshi Ohkawara $^{a}$ Hiroshi Takahashi ${ }^{a}$ \\ Hirotaka Mori $^{a}$ Mai Yaginuma ${ }^{a}$ Mamiko Endo ${ }^{a}$ \\ Akiko Shichishima-Nakamura ${ }^{a}$ Takahiro Sano $^{a}$ Kayo Harada-Shirado ${ }^{a}$ \\ Satoshi Kimura $^{a}$ Kazuei Ogawa $^{a}$ Yuko Hashimoto $^{b}$ Takayuki Ikezoe ${ }^{a}$ \\ aDepartment of Hematology, Fukushima Medical University, Fukushima, Japan; \\ bepartment of Diagnostic Pathology, Fukushima Medical University, Fukushima, Japan
}

\section{Keywords}

Acquired von Willebrand syndrome · Acute myeloid leukemia · Bleeding disorders ·

Coagulation · Haemostasis

\begin{abstract}
We here report a 21-year-old male who presented with acute myelomonocytic leukemia (AMML) associated with acquired von Willebrand syndrome (AVWS). To our knowledge, this is the first case of AVWS caused by AMML. In our case, following remission-induction chemotherapy combined with idarubicin and cytarabine, the patient showed remarkable improvement of bleeding symptoms due to AVWS. Moreover, after an allogeneic stem cell transplantation from a sibling donor, both AMML and AVWS maintain complete remission.
\end{abstract}

\section{Introduction}

Von Willebrand disease (VWD) is a hereditary disease caused by deficiency or dysfunction of von Willebrand factor (VWF) [1]. VWD is classified into several types: type 1 characterized by reduced VWF antigen (VWF:Ag), type 2 by qualitative impairment in the synthesis of VWF, and type 3 by a complete loss of VWF [2]. Additionally, type 2 is further divided into 4 subtypes: type $2 \mathrm{~A}$ is characterized by selective loss of high-molecular-weight (HMW) VWF multimers; type $2 \mathrm{~B}$ shows increased agglutination due to an increased binding affinity of VWF to glycoprotein Ib (GPIb), also known as CD42, a component of the GPIb-V-IX complex on the platelet surface; type $2 \mathrm{M}$ shows a decreased binding affinity of VWF to platelets without selective 
Fig. 1. Bone marrow smear at initial presentation was consistent with AMML FAB M4. May-Giemsa staining. Magnification, $\times 400$.

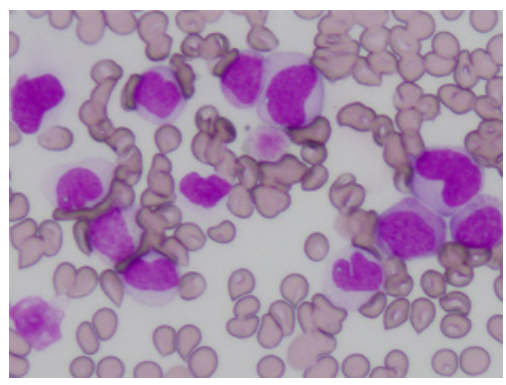

reduction of HMW VWF multimers; and type $2 \mathrm{~N}$ shows a decreased binding affinity for factor VIII (FVIII). Acquired von Willebrand syndrome (AVWS), which occurs even without genetic mutations, is a rare bleeding disorder caused by a deficiency or dysfunction of VWF [3]. The detection of inhibitory autoantibodies directed against VWF or the FVIII/VWF complex occurs in $<20 \%$ of AVWS cases [4]. The common nonimmune etiology of AVWS has been reported to include hypothyroidism, Wilms' tumor, or congenital heart defects, and the use of various drugs [5]. With regard to hematologic disorders, AVWS has been reported to be often associated with lymphoproliferative diseases such as multiple myeloma and malignant lymphoma, as well as myeloproliferative neoplasms such as essential thrombocythemia and polycythemia vera [4]. Although some cases of AVWS associated with hematological malignancies have previously been reported, the co-occurrence of AVWS with acute myelomonocytic leukemia (AMML) has never been reported.

Here, we describe a patient who had AMML associated with AVWS. To our knowledge, this is the first detailed description of AVWS associated with AMML. Interestingly, the patient showed remarkable improvement of AVWS after successful intensive chemotherapy for AMML.

\section{Case Report/Case Presentation}

A 21-year-old male was referred to our hospital for remission-induction chemotherapy for AMML. On physical examination, he was febrile with a temperature of $38.3^{\circ} \mathrm{C}$, but the rest of his vital signs were normal. He had no purpura or mucosal bleeding. Erythematous swelling on his right buttock, a few centimeters in diameter, was noted, which was considered to be cellulitis and a possible cause of fever. The patient's past and family history was negative for hemorrhagic diathesis. Laboratory tests revealed bicytopenia in peripheral blood (white blood cell count $4,300 / \mu \mathrm{L}$, hemoglobin $8.2 \mathrm{~g} / \mathrm{dL}$, and platelet count $4.2 \times 10^{9} / \mathrm{L}$ ), and $6 \%$ elevated peripheral blood blasts and showed prolongation of activated partial thromboplastin time (APTT) at $59.9 \mathrm{~s}$. Liver function, renal function, and electrolytes were normal. Bone marrow aspirate showed hypercellular marrow with 51.6\% blasts, most of which were positive for myeloperoxidase with partial monocyte-lineage differentiation, thereby establishing a diagnosis of AMML, or M4 according to the French-American-British classification (Fig. 1). Cytogenetic analysis revealed normal male karyotype, and recurrent genetic abnormalities were not detected on a multiplex quantitative polymerase chain reaction.

A central venous catheter (CVC) was inserted into his right internal jugular vein under an ultrasound-guided procedure for chemotherapy administration. After that, bleeding from the CVC insertion site persisted despite manual compression, and anemia progressed. Ultrasonography confirmed that the CVC was placed exactly in his right internal jugular vein, and there was no evident injury to the surrounding tissue or adjacent vasculature (Fig. 2).

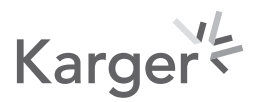




\section{Case Reports in Oncology}
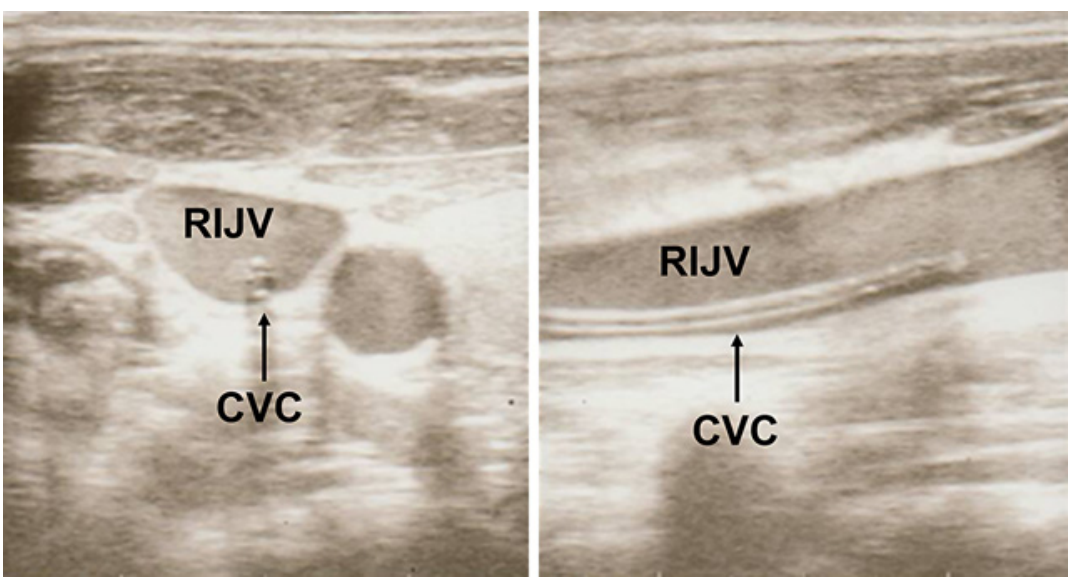

Fig. 2. Ultrasonography of the right neck in transverse (upper) and longitudinal (bottom) view. A CVC was properly inserted in the RIJV. CVC, central venous catheter; RIJV, right internal jugular vein.

Table 1. Coagulation activity tests on admission and after successful chemotherapy

\begin{tabular}{lcc}
\hline & On admission & After chemotherapy (day 40) \\
\hline PT-INR & 1.32 & 1.19 \\
APTT, s & 59.9 & 37.9 \\
FVIII, \% & 12.9 & 119.5 \\
FIX, \% & 44.2 & - \\
FXI, \% & 37.3 & - \\
FXII, \% & 30.2 & - \\
FXIII, \% & 86.3 & - \\
VWF:Ag, \% & 35 & 139 \\
VWF:RCo, \% & $<6$ & 113 \\
FVIII inhibitor, BU/mL & $<1$ & - \\
\hline \multicolumn{2}{c}{ APTT, activated partial thromboplastin time. } \\
\hline
\end{tabular}

The patient required frequent dressing changes due to persistent bleeding. On the 2nd hospital day, remission-induction chemotherapy was begun combining idarubicin $\left(12 \mathrm{mg} / \mathrm{m}^{2}\right.$ day $1-3)$ and cytarabine $\left(100 \mathrm{mg} / \mathrm{m}^{2}\right.$ day $\left.1-7\right)$. Since bleeding and hematoma around the cerebral CVC insertion site were exacerbated, the CVC was removed from his right neck and inserted into the right femoral vein. However, bleeding from the femoral CVC insertion site in the right thigh occurred again. Repeated blood coagulation test showed no evidence of uncontrolled disseminated intravascular coagulation, which often causes hemorrhagic diathesis in leukemia patients. Based on these clinical and laboratory findings, the patient was thought to have an acquired bleeding disorder. Critical laboratory findings included a VWF:Ag level of 35\%, a ristocetin cofactor activity (VWF:RCo) $<6 \%$ (below detection limit), and a FVIII activity of $12.6 \%$ (Table 1). APTT mixing study showed deficiencies of coagulation factors, and the APTT did not normalize 2-h incubation after mixing of patient's plasma with normal pooled plasma, indicating that this phenomenon was not mediated by inhibitory antibodies against VWF (Fig. 3). Moreover, decreased VWF levels were not deemed to be hereditary because none of his parents, 2 senior sisters, and a senior brother showed APTT prolongation, low VWF:Ag, or low VWF:RCo (data not shown). Based on these findings, a diagnosis of AVWS was made. 
Fig. 3. APTT mixing study showed deficiencies of coagulation factors, but not inhibitory antibodies against VWF. APTT, activated partial thromboplastin time.

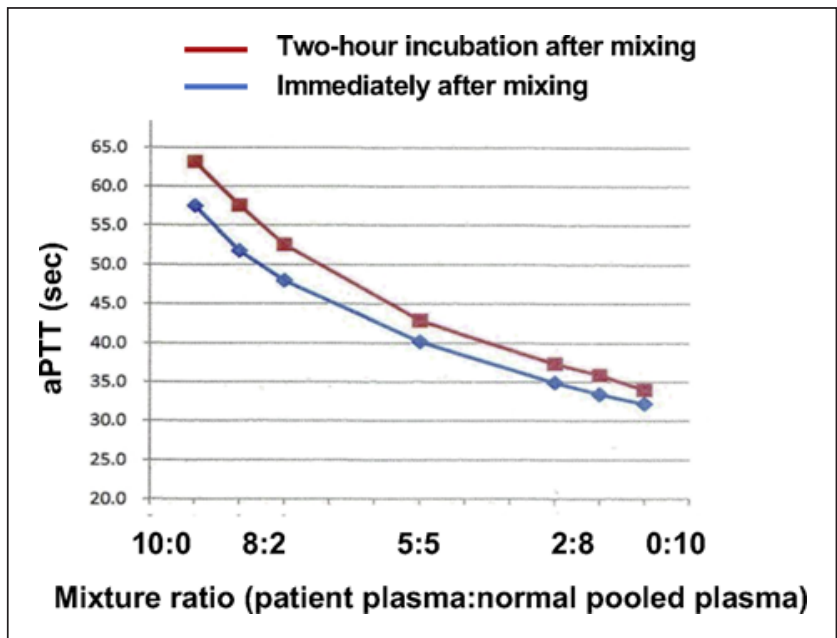

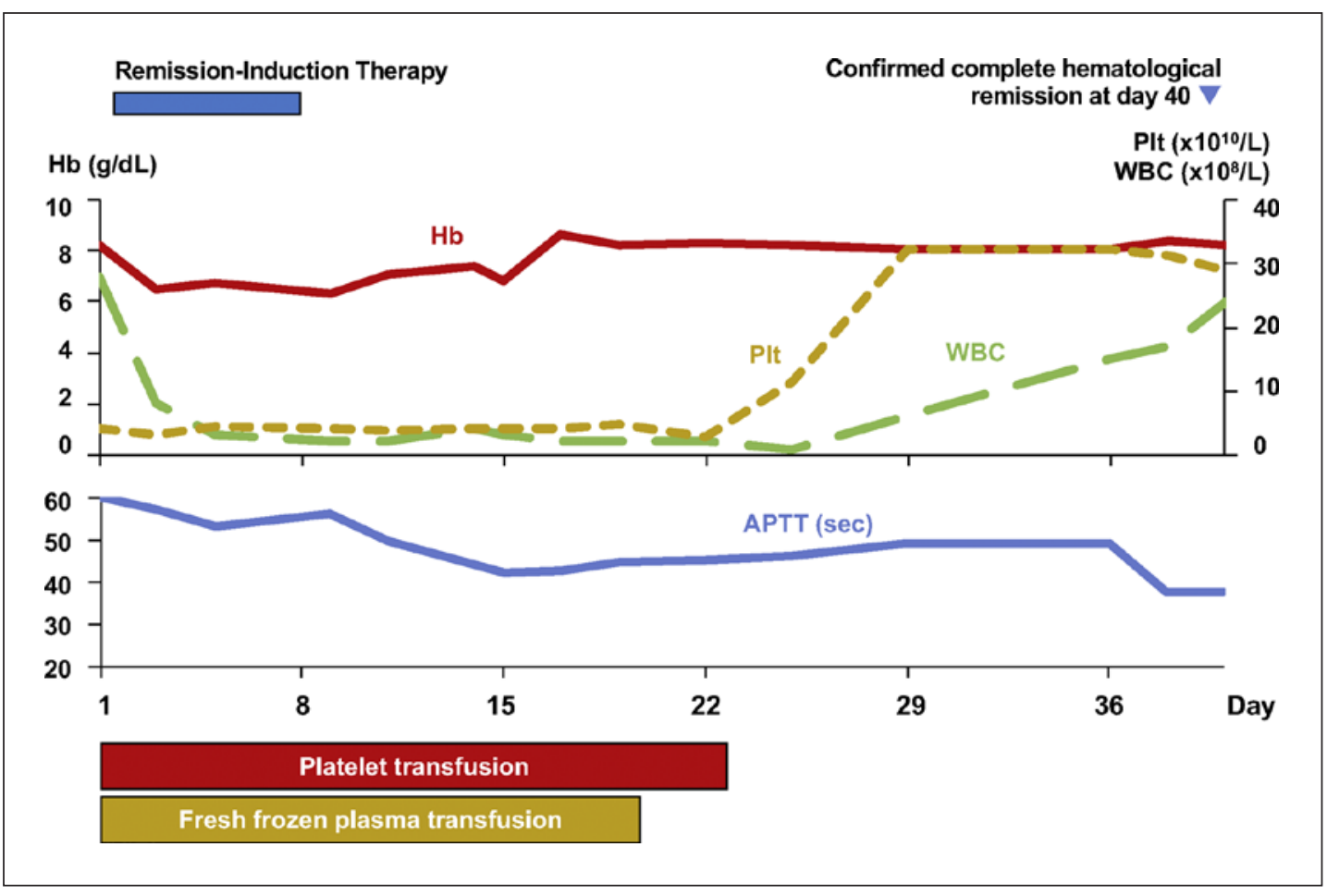

Fig. 4. Clinical course.

At this point, it was unclear whether or not AVWS was associated with AMML. He was administered fresh frozen plasma and platelet transfusions, which did not improve his bleeding severity. However, his bleeding showed remarkable improvement after intensive remission-induction chemotherapy. Bone marrow aspirate after remission-induction therapy confirmed complete hematological remission of AMML (Fig. 4). Concomitantly, VWF:Ag and VWF:RCo also recovered to 139 and 113\%, respectively (Table 1). Following 3 cycles of consolidation chemotherapy for AMML, the patient received an allogeneic stem cell transplant from a sibling donor. He is still in remission for both AMML and AVWS, and no remarkable bleeding episodes have been observed afterward. 


\section{Case Reports in Oncology}
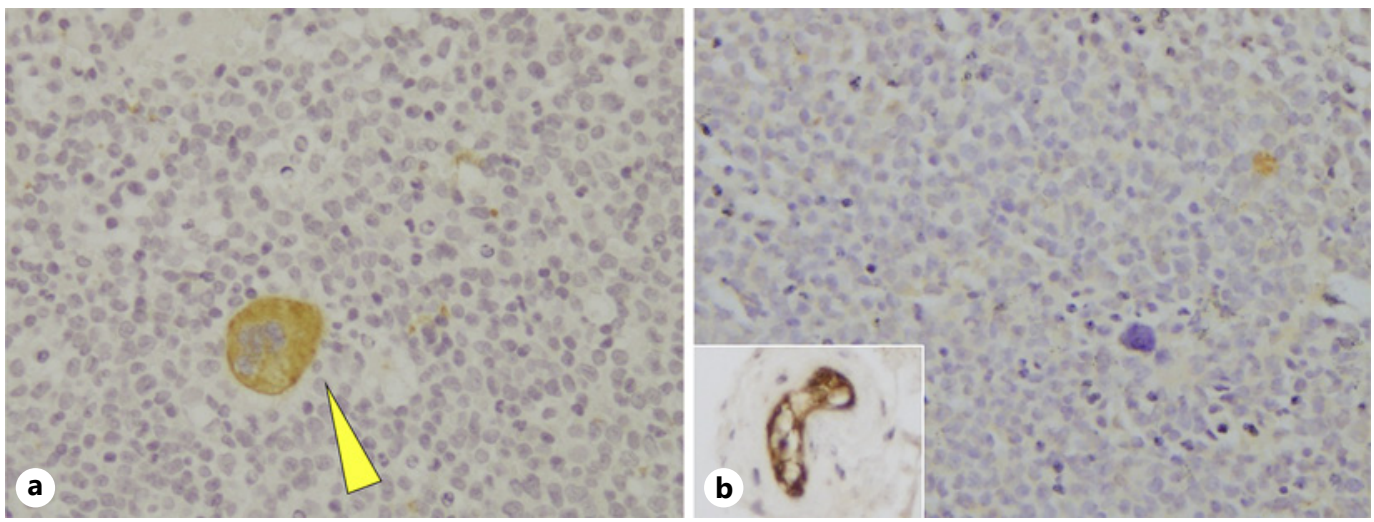

Fig. 5. Immunohistochemistry of leukemic cells of the bone marrow. The expressions of GPIb (a) and VWF (b) were not detected in the leukemic cells. a Arrowheads indicate megakaryocytes positive for GPIb. b Endothelial cells of a bone marrow biopsy specimen, in the inset, which was obtained from an unrelated patient with chronic myeloid leukemia in complete remission, serve as positive control. Magnification, $\times 200$.

\section{Discussion/Conclusion}

Diagnosis of AVWS is difficult, especially when complicated by underlying medical conditions, as there are no definitive diagnostic criteria, nor a single test sufficient to prove or exclude AVWS [2,6]. AVWS type 1 or 3 in children with Wilms' tumor disappears after successful chemotherapy or tumor resection, but the mechanism of the VWF deficiency remains unknown [4]. AVWS type 2 in patients with thrombocythemia of various myeloproliferative disorders is caused by increased proteolysis of large VWF multimers at increasing platelet counts to above $1,000 \times 10^{9} / \mathrm{L}$ [4].

VWF:RCo/VWF:Ag ratio is known to be useful in the classification of congenital VWD since the disparity between VWF:RCo and VWF:Ag reflects structural abnormalities and mutations within specific regions of VWF gene [6]. In our case, VWF:RCo markedly decreased, while VWF:Ag was disproportionately preserved (Table 1), which made VWF:RCo/VWF:Ag ratio $<0.6$. In addition, APTT mixing study indicated deficiencies of coagulation factors as the cause of coagulopathy (Fig. 3). These results collectively showed qualitative impairment in the synthesis of VWF. Thus the etiology of AVWS may be due to the depletion of HMW VWF multimers. Although FVIII activity also modestly decreased, FVIII inhibitor was not detected in our case, and such observations have occasionally been reported elsewhere [7].

Several mechanisms underlying AVWS have been proposed so far [8]: (1) drug- or autoantibody-mediated interference to VWF activity, (2) autoantibody-mediated clearance of VWF from circulation, (3) adsorption to surfaces of certain cells or tissues, for example, increased platelets or tumor cells, or (4) increased shear stress and subsequent proteolysis. In our case, GPIb and VWF expressions in leukemia cells were not detected by immunohistochemical staining (Fig. 5a, b), thereby ruling out a hypothesis that adsorption of VWF to leukemia-expressing GPIb causes loss of VWF activity, a mechanism previously described in lymphoproliferative disease-associated AVWS $[9,10]$. Additionally, it is noteworthy that the underlying condition of our patient was leukemia, not lymphoid neoplasms or autoimmune disorders, which makes it less probable that autoantibody was involved in our case. Although difficult to validate, an intriguing hypothesis is that leukemic cells secreted structurally abnormal proteolytic enzymes, or proteolytic enzymes with altered physiological function, thereby interfering with HMW VWF proteolysis $[11,12]$. Indeed, the etiology of AVWS has been poorly understood. Accumulation of cases and further investigations are required.

\section{Karger'}


In conclusion, to our knowledge, this is the first detailed description of AVWS associated with AMML. Clinicians should be aware of the possibility that, in leukemia patients, not only thrombocytopenia and disseminated intravascular coagulation but also AVWS can cause severe hemorrhagic diathesis [13]. Extensive investigation of coagulation is warranted in such cases, especially when complicated by prolonged APTT.

\section{Statement of Ethics}

Written informed consent was obtained from the patient for publication of this case report and any accompanying images.

\section{Conflict of Interest Statement}

The authors have no conflicts of interest to declare.

\section{Funding Sources}

The authors did not receive any funding.

\section{Author Contributions}

M.F., H.O., and T.I. wrote the manuscript. T.I. supervised the project. All the authors have participated in this case.

\section{References}

1 Ng C, Motto DG, Di Paola J. Diagnostic approach to von Willebrand disease. Blood. 2015;125(13):2029-37.

2 Gadisseur A, Hermans C, Berneman Z, Schroyens W, Deckmyn H, Michiels JJ. Laboratory diagnosis and molecular classification of von Willebrand disease. Acta Haematol. 2009;121(2-3):71-84.

3 Tiede A, Rand JH, Budde U, Ganser A, Federici AB. How i treat the acquired von Willebrand syndrome. Blood. 2011;117(25):6777-85.

4 Sucker C, Michiels JJ, Zotz RB. Causes, etiology and diagnosis of acquired von Willebrand disease: a prospective diagnostic workup to establish the most effective therapeutic strategies. Acta Haematol. 2009;121(2-3):17782.

5 Shetty S, Kasatkar P, Ghosh K. Pathophysiology of acquired von Willebrand disease: a concise review. Eur J Haematol. 2011;87(2):99-106.

6 Stufano F, Boscarino M, Bucciarelli P, Baronciani L, Maino A, Cozzi G, et al. Evaluation of the utility of von Willebrand factor propeptide in the differential diagnosis of von Willebrand disease and acquired von Willebrand syndrome. Semin Thromb Hemost. 2019;45(1):36-42.

7 Koyama T, Fujimoto K, Shima M. Acquired von Willebrand syndrome associated with Hashimoto's thyroiditis and subcutaneous mucosa-associated lymphoid tissue lymphoma. Intern Med. 2013;52(23):2661-3.

8 Federici AB. Acquired von Willebrand syndrome: is it an extremely rare disorder or do we see only the tip of the iceberg? J Thromb Haemost. 2008;6(4):565-8.

9 Richard C, Cuadrado MA, Prieto M, Batlle J, López Fernández MF, Rodriguez Salazar ML, et al. Acquired von Willebrand disease in multiple myeloma secondary to absorption of von Willebrand factor by plasma cells. Am J Hematol. 1990;35(2):114-7.

10 Tefferi A, Hanson CA, Kurtin PJ, Katzmann JA, Dalton RJ, Nichols WL. Acquired von Willebrand's disease due to aberrant expression of platelet glycoprotein Ib by marginal zone lymphoma cells. Br J Haematol. 1997; 96(4):850-3.

11 Federici AB, Berkowitz SD, Lattuada A, Mannucci PM. Degradation of von Willebrand factor in patients with acquired clinical conditions in which there is heightened proteolysis. Blood. 1993;81(3):720-5. 


\section{Case Reports in Oncology}

12 Federici AB. Clinical and laboratory diagnosis of VWD. Hematology Am Soc Hematol Educ Program. 2014; 2014(1):524-30.

13 Leinoe EB, Hoffmann MH, Kjaersgaard E, Nielsen JD, Bergmann OJ, Klausen TW, et al. Prediction of haemorrhage in the early stage of acute myeloid leukaemia by flow cytometric analysis of platelet function. $\mathrm{Br} \mathrm{J}$ Haematol. 2005;128(4):526-32. 